\title{
Alimentos saludables y no saludables en la opinión de adolescentes de las escuelas públicas y privadas en la capital del nordeste brasileño
}

\section{Opinions on healthy and unhealthy foods among adolescentes from public and private schools in a Northeastern Brazilian capital city}

\section{RESUMEN}

Objetivo. Analizar la opinión de los adolescentes sobre alimentos saludables y no saludables. Estudio transversal con adolescentes de escuelas públicas y privadas. Métodos. La muestra se estratificó en tres etapas: escuela, clase y alumno, resultando en 1507 adolescentes estudiados. Los adolescentes respondieron sobre alimentos saludables y no saludables. Resultados: 995 adolescentes respondieron al cuestionario de forma completa. Los adolescentes concordaron con los beneficios de las frutas para la salud. Hubo discordancia entre las respuestas de los estudiantes de escuelas públicas y privadas sobre los maleficios de los saladitos ultraprocesados y las papas fritas para la salud; estudiantes de la red privada de enseñanza, así como del sexo femenino concordaron que las gaseosas ayudan a engordar y provocan obesidad, mientras que los de la red pública discreparon; adolescentes de escuelas privadas, de sexo femenino y grupo de edad de 15 a 17 años discreparon que carnes rojas y grasas protegen contra enfermedades del corazón. Conclusión: Los adolescentes de las escuelas de la red privada de enseñanza parecen presentar una mejor percepción acerca de los refrescos y carnes y sus maleficios a la salud, como aquellos que presentaron la mayor edad y del sexo femenino.

Palabras clave: Adolescente; Alimentos integrales; Escuelas; Opinión.

\footnotetext{
ABSTRACT

Objective: To analyze adolescents' opinion on healthy and unhealthy foods. We conducted a cross-sectional study involving adolescents from public and private schools. Methods: The sample was stratified in three stages: school, class and student, resulting in 1507 surveyed adolescents. The adolescents answered questions on healthy and unhealthy foods. Results: A total of 995 adolescents answered the questionnaire and were considered for analysis. By and large, adolescents agreed on the benefits of fruits for health. There was disagreement between responses of public versus private school students with regard to the health risks of processed salty snacks and French fries; private school students, as well as females, agreed that soft
}

Marize Melo dos Santos ${ }^{1}$, Alana Rafaela da Silva Moura', Thalita Braga Barros Abreu', Adriana de Azevedo Paiva', Cecília Maria Resende Gonçalves de Carvalho't.

1. Departamento de Nutrición, Universidad Federal de Piauí.

Dirigir correspondencia a: Marize Melo dos Santos Campus Universitario Ministro Petronio Portella, Departamento de Nutrición, Bloque 13. Barrio Ininga, CEP: 64049-550. Teléfono: +55 86-32155863. E-mail: marizesantos@ufpi.edu.br

Este trabajo fue recibido el 10 de noviembre de 2017. Aceptado con modificaciones: 04 de julio de 2018. Aceptado para ser publicado: 09 de agosto de 2019.

drinks contribute to weight gain and obesity, while those belonging to the public schools disagreed; adolescents from private schools, female and adolescents aged 15 to 17 years disagreed that red and fatty meats protect against heart diseases. Conclusion: Adolescents from private versus public schools, females compared to males, and who were older demonstrated a better understanding of the negative health effects of soft drinks and meat consumption Keywords: Adolescent; Healthy Foods; Opinion; Schools.

\section{INTRODUCCIÓN}

La adolescencia es el período comprendido entre los 10 a 19 años de edad, que se caracteriza por la transición entre la infancia y la edad adulta y está caracterizada por 
cambios biológicos, psicológicos y sociales'. Teniendo en cuenta estos aspectos, la nutrición juega un papel fundamental, pues delimita condiciones favorables al crecimiento y desarrollo del individuo ${ }^{2}$.

Es importante el seguimiento y monitoreo de la calidad de los alimentos que se consumen durante la fase de la adolescencia. De acuerdo con Pearson et al. ${ }^{3}$ en esa fase, además de la rutina alimentaria interferir en el crecimiento y desarrollo, porque el consumo de frutas y vegetales a corto o largo plazo, que trae beneficios de protección a la salud ha declinado, sin embargo, el consumo de alimentos que causan adiposidad corporal es activamente adoptados durante esa edad.

La alimentación en la adolescencia ha cobrado gran atención, principalmente, por la relación entre los hábitos alimentarios inadecuados y el desarrollo de determinadas enfermedades, por lo que conocer las representaciones relacionadas con la alimentación de esos individuos es fundamental para el desarrollo de acciones de promoción y de educación en salud, de modo que se alcance efectivamente el grupo y movilicen cambios de hábitos².

La familia se caracteriza como el primer grupo social con influencia directa sobre la alimentación de adolescentes, ya que es exactamente en el ambiente familiar que los niños y adolescentes tienen su primer contacto con la alimentación, y siendo la responsable de la compra y preparación de los alimentos a domicilio, tiene gran oportunidad de promover el incentivo a la alimentación sana ${ }^{4,5}$.

Por otro lado, los medios tienen gran influencia en el comportamiento alimentario, pues actúa diseminando informaciones sobre los alimentos, estimulando el consumo de alimentos sanos o no ${ }^{6}$. En esta fase de la vida, la adquisición de comportamientos no saludables puede llevar a la exposición de situaciones de riesgo, de las cuales pueden venir serias consecuencias en el futuro?

La escuela también influye en la alimentación de los adolescentes, pues en ese espacio el alumno permanece por un largo período y es expuesto a alimentos y preparaciones que pueden crear cambios en sus hábitos alimenticios, ya que en ese ambiente hay incentivo de la educación en salud, escuelas que presentan la merienda escolar. En la escuela el adolescente puede recibir interferencias alimentarias de los estímulos presentes en el ambiente escolar, así como del grupo social que está inserto, pues la alimentación en la adolescencia generalmente está relacionada a una identidad grupal ${ }^{8}$.

En general, el consumo alimentario de los adolescentes se caracteriza por la preferencia de alimentos con alto contenido energético, tales como productos con alto contenido de grasa saturada, colesterol, sodio y carbohidratos refinados 9 , algunos estudios nacionales ${ }^{10,11,12} \mathrm{e}$ internacionales ${ }^{13,14}$ confirman el creciente consumo de esos alimentos por ese público. A este respecto, la dieta de los adolescentes es representada muchas veces por salados, saladitos, alimentos fritos de origen animal, así como bebidas con adición de azúcar, por lo tanto, alimentos visualmente atractivos ${ }^{9}$.
Ante el escenario actual sobre la alimentación, el estudio en cuestión analizó las preferencias de adolescentes pertenecientes a las escuelas públicas y privadas de Teresina, en relación a los alimentos saludables y no saludables.

\section{MÉTODOS \\ Delineamiento y población del estudio}

Se trata de un estudio transversal, multicéntrico, nacional, en ámbito escolar, utilizando el banco de datos del proyecto suplementario al Estudio de Riesgos Cardiovasculares en Adolescentes (ERICA), realizado en el período de 2013 a 2014, con adolescentes, de ambos sexos, de 12 a 17 años, de escuelas públicas y privadas de Teresina-PI, desarrollado por la Universidad Federal de Río de Janeiro/Instituto de Estudios en Salud Colectiva y Universidad Federal de Piauí/ Departamento de Nutrición. La presente propuesta analizó datos relativos a las actitudes alimenticias saludables y no saludables.

\section{Muestras}

La muestra se estratificó en tres etapas: escuela, clase y alumno, incluyendo tres clases por escuela, resultando en 1507 adolescentes que participaron del estudio ERICA, en 30 escuelas públicas y privadas, de las zonas distritales norte, sur, este y sureste de Teresina, los cuales respondieron a un cuestionario referente a alimentos saludables y no saludables. Del total de encuestados, sólo 995 adolescentes respondieron al cuestionario de forma completa.

\section{Plan de recolección y análisis de datos}

Los alumnos fueron orientados por profesionales de campo debidamente entrenados a responder un cuestionario de nueve afirmaciones sobre alimentos saludables y no saludables, mediante el cual se analizaron las actitudes alimentarias, a través de las opiniones concordantes o discordantes, comparando los adolescentes de escuela pública y privadas, por sexo y grupo de edad. Las afirmaciones se dispusieron de la siguiente manera: a) Las frutas son fuentes de vitaminas y minerales, hacen bien la salud y ayudan a prevenir enfermedades; b) Los saladitos ultraprocesados (bocados) y las papas fritas promueven el bienestar y consecuentemente hacen bien para la salud; c) Los refrescos ayudan a engordar y provocan obesidad; d) Las carnes rojas y grasas protegen contra las enfermedades del corazón;

Los datos se dispusieron en el software STATA versión 14.0. Se consideró $p<0,05$, para la significancia estadística. Se utilizó la prueba de asociación de Chi-cuadrado para todas las asociaciones entre las variables.

\section{Consideraciones éticas}

El protocolo del estudio fue sometido y aprobado por el Comité de la Universidad Federal de Piauí bajo el número 406.353. Después de la aclaración de los objetivos de la investigación, todos los participantes firmaron término de asentimiento. 


\section{RESULTADOS}

Del total de la muestra $(n=1507)$, se evaluaron $995(66 \%)$, siendo $73,6 \%$ de escuelas públicas y $56,6 \%$ del sexo femenino, con una media de edad 14,7 años ( $\mathrm{DP}=1,5$ años).

Los resultados muestran las opiniones de los adolescentes pertenecientes a escuelas públicas y privadas de Teresina, capital de Piauí, Nordeste de Brasil, sobre alimentación sana y no saludable.

No se encontraron diferencias significativas en las respuestas de los estudiantes, según sexo, tipo de escuela y edad ( $p>0,05$ ), según concordancia/discordancia con la afirmación "Las frutas son fuentes de vitaminas y minerales, hacen bien para la salud y ayudan a prevenir enfermedades" (Tabla 1).

En la Tabla 2, las respuestas según concordancia/discordancia con la afirmativa "Los saladitos ultraprocesados (bocados) y las papas fritas promueven el bienestar y consecuentemente hacen

Tabla 1. Distribución de los adolescentes según concordancia/ desacuerdo con la afirmación "Las frutas son fuentes de vitaminas y minerales, hacen bien a la salud y ayudan a prevenir enfermedades". Teresina, 2014

\begin{tabular}{|lccccc|}
\hline Variables & $\begin{array}{c}\text { Estoy de acuerdo } \\
\mathbf{n}\end{array}$ & $\begin{array}{c}\text { No estoy de acuerdo } \\
\mathbf{n}\end{array}$ & $*_{\mathbf{p}}$ \\
& & & & & \\
\hline $\begin{array}{l}\text { Tipo de escuela } \\
\quad \text { Público }\end{array}$ & 721 & 98,5 & 11 & 1,5 & 0,363 \\
$\quad$ Privada & 261 & 99,2 & 2 & 0,8 & \\
Sexo & & & & & \\
$\quad$ Mujer & 558 & 99,1 & 5 & 0,9 & 0,185 \\
$\quad$ Hombre & 424 & 98,1 & 8 & 1,85 & \\
Edad (años) & & & & & \\
12-14 & 457 & 98,1 & 9 & 1,9 & 0,103 \\
$15-17$ & 525 & 99,2 & 4 & 0,8 & \\
\hline
\end{tabular}

* Prueba de asociación de Chi-cuadrado.

Tabla 3.Distribución de los adolescentes según concordancia/ desacuerdo con la afirmación "Gaseosas ayudan a engordar y provocan obesidad". Teresina, 2014.

\begin{tabular}{|c|c|c|c|c|c|}
\hline \multirow[t]{2}{*}{ Variables } & \multicolumn{2}{|c|}{ Estoy de acuerdo } & \multicolumn{2}{|c|}{ No estoy de acuerdo } & \multirow[t]{2}{*}{$* \mathbf{P}$} \\
\hline & n & $\%$ & $\mathbf{n}$ & $\%$ & \\
\hline \multicolumn{6}{|l|}{ Tipo de escuela } \\
\hline Público & 587 & 80,2 & 145 & 19,8 & $<0,001$ \\
\hline Privada & 245 & 98,2 & 18 & 6,8 & \\
\hline \multicolumn{6}{|l|}{ Sexo } \\
\hline Mujer & 484 & 86,0 & 79 & 14,0 & 0,022 \\
\hline Hombre & 348 & 80,6 & 84 & 19,4 & \\
\hline \multicolumn{6}{|l|}{ Edad (años) } \\
\hline $12-14$ & 386 & 82,8 & 80 & 17,2 & 0,530 \\
\hline $15-17$ & 446 & 83,5 & 83 & 15,7 & \\
\hline
\end{tabular}

*Prueba de asociación de Chi-cuadrado. bien a la salud" hubo mayor porcentaje de discordancia para aquellos de escuelas privadas $(75,3 \% ; p=0,002)$ y de mayor edad $(70,4 \% ; p=0,023)$. Sin embargo, es importante destacar que entre el $25 \%$ a $36,0 \%$ de los estudiantes, para todas las variables, concordaron con esa afirmación.

En la Tabla 3, la mayoría de los adolescentes (más del 80\%) respondieron concordante para la afirmación "Las gaseosas ayudan a engordar y provocan obesidad". Sin embargo, casi el $20 \%$ de los estudiantes de las escuelas públicas y los hombres discrepaban de esta afirmación.

En cuanto a la afirmación "Las carnes rojas y grasas protegen contra las enfermedades en el corazón", más del $60 \%$ de los adolescentes demostraron no tener conocimiento sobre ese asunto, siendo significativa para las categorías, tipo de escuela, sexo y edad $(p<0,01)$.

Tabla 2. Distribución de los adolescentes según concordancia/ desacuerdo con la afirmación "Los saladitos ultraprocesados y las papas fritas promueven el bienestar y consecuentemente hacen bien la salud". Teresina, 2014.

\begin{tabular}{|c|c|c|c|c|c|}
\hline \multirow[t]{2}{*}{ Variables } & \multicolumn{2}{|c|}{ Estoy de acuerdo } & \multicolumn{2}{|c|}{ No estoy de acuerdo } & \multirow[t]{2}{*}{$* \mathbf{p}$} \\
\hline & & $\%$ & & & \\
\hline \multicolumn{6}{|l|}{ Tipo de escuela } \\
\hline Público & 258 & 35,2 & 474 & 64,8 & 0,002 \\
\hline Privada & 65 & 24,7 & 198 & 75,3 & \\
\hline \multicolumn{6}{|l|}{ Sexo } \\
\hline Mujer & 171 & 30,4 & 392 & 69,6 & 0,108 \\
\hline Hombre & 152 & 35,2 & 280 & 64,8 & \\
\hline \multicolumn{6}{|l|}{ Edad (años) } \\
\hline $12-14$ & 168 & 36,0 & 298 & 64,0 & 0,023 \\
\hline $15-17$ & 155 & 29,3 & 374 & 70,7 & \\
\hline
\end{tabular}

* Prueba de asociación de Chi-cuadrado.

Tabla 4. Distribución de los adolescentes según concordancia/ desacuerdo con la afirmación "Carnes rojas y grasas protegen contra las enfermedades del corazón". Teresina, 2014.

\begin{tabular}{|c|c|c|c|c|c|}
\hline \multirow[t]{2}{*}{ Variables } & \multicolumn{2}{|c|}{ Estoy de acuerdo } & \multicolumn{2}{|c|}{ No estoy de acuerdo } & \multirow[t]{2}{*}{ P* } \\
\hline & $\mathbf{N}$ & $\%$ & $\mathbf{n}$ & $\%$ & \\
\hline \multicolumn{6}{|c|}{ Tipo de escuela } \\
\hline Público & 252 & 34,4 & 480 & 65,6 & 0,001 \\
\hline Privada & 61 & 23,2 & 202 & 76,8 & \\
\hline \multicolumn{6}{|l|}{ Sexo } \\
\hline Mujer & 148 & 26,3 & 415 & 73,7 & $<0,001$ \\
\hline Hombre & 165 & 38,2 & 267 & 61,8 & \\
\hline \multicolumn{6}{|l|}{ Edad (años) } \\
\hline $12-14$ & 165 & 35,4 & 301 & 64,6 & 0,012 \\
\hline $15-17$ & 148 & 28,0 & 381 & 72,0 & \\
\hline
\end{tabular}

*Prueba de asociación de Chi-cuadrado. 


\section{DISCUSIÓN}

La mayor diferencia en la caracterización de los 995 participantes del estudio se refiere al número de adolescentes de escuelas públicas en relación a las privadas, en las opiniones sobre alimentos saludables y no saludables. Las demás características, sexo y grupo de edad, poseen diferencias, pero en menor destaque, significativas o no.

Los estudios sobre adolescentes son relevantes ya que es una fase repleta de transformaciones y una de ellas se refiere al contexto de la alimentación. Con base en ello, conocer las preferencias alimentarias de los adolescentes se hace importante para contribuir al desarrollo sano de los mismos.

La mayoría de los estudiantes, independientemente de la escuela, el sexo y el grupo de edad concuerdan que las frutas son fuentes de vitaminas y minerales, hacen bien a la salud y ayudan a prevenir enfermedades. Esto puede haber sido debido a la mayor difusión de información sobre la importancia de este tipo de alimento en los días actuales.

Los hallazgos antecedentes, en los años 2008-2009, provenientes de las Encuestas de Presupuestos Familiares (POFs) del Instituto Brasileño de Geografía y Estadística (IBGE), mostraban que el patrón alimentario del brasileño se caracterizaba por la persistencia de algunos hábitos alimentarios tradicionales, como el consumo de frutas y hortalizas, que son alimentos saludables. Sin embargo, se observa una creciente y preocupante participación de productos ultra-procesados en la dieta de ese público ${ }^{15}$.

Silva et al. ${ }^{16}$ revelaron que factores facilitadores de la adhesión de los adolescentes a la alimentación sana se relacionaron con el gusto de algunos alimentos saludables, el acceso y la disponibilidad a estos alimentos, el miedo a engordar, el ambiente familiar, así como la escuela por medio de prácticas educativas alimentarias. El conocimiento sobre alimentación sana referido por los adolescentes estuvo asociado con la ingestión adecuada de verduras, legumbres y frutas por prevenir la instalación de una serie de agravios a la salud.

Los mismos autores mostraron que un punto relevante relatado por los adolescentes se refiere al papel de la escuela en el proceso de formación de hábitos alimentarios saludables a través del desarrollo de prácticas educativas alimentarias y nutricionales que abordan informaciones sobre ejemplos de alimentos saludables, la importancia de la realización de elecciones alimentarias saludables y a intervalos regulares, y algunos beneficios de la alimentación saludable para la salud ${ }^{16}$.

Tavares et al. ${ }^{17}$ realizaron una investigación con adolescentes de escuelas públicas y privadas de la ciudad de Río de Janeiro y mostraron que no hubo diferencia en la proporción de adolescentes con consumo regular de alimentos marcadores de la alimentación sana, tales como frutas frescas o ensalada de fruta.

La investigación sobre el uso de actividades educativas promotoras de alimentación sana teniendo como base el escenario de las escuelas públicas y privadas de la capital de Brasil, Silva et al. ${ }^{18}$, mostraron que la implementación de sitios específicos para divulgar informaciones sobre salud y alimentación, en las escuelas públicas hubo una asociación significativa con el uso permanente de estos sitios en escuelas privadas.

Los mismos autores, al analizar la presencia de vendedores ambulantes y tiendas locales cerca de la escuela (a unos 50 metros de la entrada) encontraron una mayor concentración de éstos cerca de las escuelas públicas. Además, escuelas privadas poseían la presencia de un lugar para clases de culinaria, lo que ayuda en la percepción favorable de los estudiantes sobre alimentos saludables ${ }^{18}$.

Souza et al. ${ }^{19}$ estudiaron adolescentes de la red pública de enseñanza y verificaron que el tema más frecuente entre ese público, independiente del sexo, fue el alto consumo de salados fritos, galletas rellenas, chocolate y de caramelos, diariamente. Estos alimentos, así como los saladitos ultraprocesados (bocados) y papas fritas, poseen alta densidad calórica, y fueron vistos por una cantidad significativa por los estudiantes de escuelas públicas como promotores del bienestar y beneficiosos para la salud.

En cuanto a la edad, Oliveira et al. ${ }^{20}$ evaluaron el uso de televisión y consumo de comidas y aperitivos por adolescentes brasileños. La mayoría de los adolescentes se refirió a consumir aperitivos frente a la televisión a veces, y aproximadamente el $40,0 \%$ casi siempre o siempre adoptan tal práctica. Este hábito fue más prevalente en adolescentes con edad entre 12 y 14 años. Una posible explicación es que, con el aumento de la edad, el adolescente, permanece menos tiempo frente a las pantallas, debido a algunos factores, como culturales, que los llevan a ejercer otras actividades.

Esto muestra que los adolescentes con menor edad parecen estar más expuestos a prácticas no saludables, lo que puede influir en las opiniones presentadas por estos individuos, como se observa en este trabajo.

Es posible observar que probablemente en las escuelas privadas los adolescentes tienen una mejor percepción de los efectos maléficos causados por alimentos no saludables, a ejemplo de las gaseosas, hecho que puede impulsar las preferencias alimentarias negativas en cuanto a las bebidas azucaradas. Se ha visto también que los adolescentes varones parecen poseer hábitos alimentarios más inadecuados que las niñas por posiblemente presentar actitudes no saludables.

Silva et al. ${ }^{18}$, verificaron que uno de los factores utilizados para la promoción de la alimentación sana entre escuelas públicas y privadas, fue la realización de reuniones para discusión de la alimentación sana con padres y funcionarios. En las escuelas públicas, el número promedio de reuniones realizadas anualmente para discutir la alimentación sana con los padres y funcionarios fue de una para cada grupo. En las escuelas privadas, estas fueron 2,4 reuniones/año con los padres y 3,6 con los funcionarios. Las sugerencias levantadas durante las reuniones con los miembros de la comunidad escolar se consideraron en la elaboración de la Política de Enseñanza en el 59\% de las escuelas.

En cuanto al sexo, Silva et al. ${ }^{21}$, realizaron un estudio 
sobre comportamientos de riesgo hecho con adolescentes en el Nordeste de Brasil y revelaron que los adolescentes del sexo masculino fueron influenciados por un número mayor de factores (consumo de refrescos, exposición al comportamiento sedentario y bajo nivel de actividad física que las mujeres (consumo de refrescos y sobrepeso/obesidad). A pesar de que el consumo de refrescos está presente en ambos sexos como factor de riesgo, los adolescentes varones han sido influenciados por más factores que las niñas, indicando probablemente, que las niñas poseen mejores hábitos.

Oliveira et al. ${ }^{20}$ verificaron que, en relación al tiempo de la pantalla, la gran parte de los adolescentes refirió pasar dos o más horas del día haciendo uso de TV, computadora y videojuego, siendo ese comportamiento más prevalente entre los niños $(74,7 \%)$. Este comportamiento ante las pantallas lleva a las personas, en general, a no prestar atención a lo que consumen y no masticar de manera adecuada.

De acuerdo con Leal et al. ${ }^{22}$, el tiempo pasado frente a las pantallas contribuye tanto al sedentarismo y al consumo excesivo de energía, pues además de encontrarse en esta situación durante horas, niños y adolescentes están más expuestos a la propaganda de alimentos no sanos y consecuentemente tienden a consumir más fast-food y refrescos que aquellos que pasan menos tiempo dedicados a la TV, lo que puede contribuir a preferencias alimentarias no saludables.

Posiblemente, el consumo adecuado de carnes rojas, principalmente, entre los adolescentes de la red privada de enseñanza, puede contribuir a la adopción de preferencias saludables en cuanto a esos alimentos, a ejemplo de estar de acuerdo que carnes rojas cuando grasas no son beneficiosas para la salud.

También se verificó que los niños parecen poseer hábitos menos saludables que las niñas en cuanto al consumo de carnes rojas y grasas, favoreciendo la formación de una opinión desfavorable en cuanto a ese tipo de alimento.

Los investigadores analizaron el consumo alimentario de escolares de las redes públicas y privadas de enseñanza en São Luís, Maranhão y verificaron el consumo adecuado de carnes tanto en la red pública como en la privada. Cuando el consumo estuvo relacionado sólo con las carnes rojas, se notó un mayor porcentaje por los estudiantes de la red privada de enseñanza ${ }^{23}$.

Schneider et al. ${ }^{24}$, evaluaron el consumo de carnes por adultos de Brasil y verificaron que el grupo de las carnes rojas, compuesto por las carnes con hueso, filete y carne picada, fue consumido por el 99,3\% de las personas en el último año, siendo el mayor consumo de carnes con hueso $(93,0 \%)$ seguida de la carne picada $(91,8 \%)$ y del filete $(87,6 \%)$. Estas carnes fueron discretamente más consumidas por hombres, pero con relación al consumo de carnes con exceso de grasa, eso fue referido por el $52,3 \%$ de los individuos, siendo el $66,7 \%$ entre los hombres.

De acuerdo con Pearson et al. ${ }^{3}$, los hábitos alimentarios adoptados durante la infancia y la adolescencia son los que probablemente perdurarán durante la fase adulta, indicando probablemente que los adolescentes de ese estudio pueden desarrollar el consumo de carnes grasas en la fase adulta también, principalmente los del sexo masculino que presentaron una relación menos sana con carnes rojas y grasas en comparación con las niñas.

En lo que se refiere al grupo de edad, es probable que con el aumento de la edad los adolescentes acaban por adquirir mayor conocimiento, pudiendo reflejarse en preferencias alimentarias adecuadas, a ejemplo de discrepar que carnes rojas y grasas protegen contra las enfermedades del corazón.

Considerando que la opinión es el "modo de ver personal" o "manifestación de las ideas individuales acerca de algo o alguien" y el conocimiento es conceptuado como "saber", "entendimiento sobre algo" ${ }^{25}$, así, la opinión pode influir el consumo y el patrón alimentario de los adolescentes, y ésta, a su vez, contribuye a la formación de preferencias alimenticias adecuadas o inadecuada.

\section{CONCLUSIÓN}

El conocimiento sobre las frutas se encuentra diseminado entre los adolescentes, contribuyendo para una buena percepción de esos alimentos independientemente de las variables analizadas.

Los adolescentes de las escuelas de la red privada de enseñanza parecen presentar una mejor percepción acerca de las gaseosas y las carnes y sus maleficios a la salud, así como a aquellos que presentaron la mayor edad y el sexo femenino.

Es importante resaltar la relevancia de estrategias nutricionales educativas en las escuelas, de forma que contribuya a la formación del conocimiento adecuado sobre los alimentos en los adolescentes, principalmente, en los que integran la red pública de enseñanza.

Agradecimiento: Universidad Federal de Río de Janeiro / Instituto de Estudios en Salud Colectiva (UFRJ / IESC)

Apoyo financiero: Fundación de Amparo a la Investigación del Estado de Piauí por el apoyo financiero para la revisión del artículo en español.

\section{BIBLIOGRAFÍA}

1. World Health Organization. Physical status: use and interpretation of anthropometry. Report of a WHO Expert Committee. Geneva; (WHO Technical Report Series, 834), 1995.

2. Silva JG, Teixeira MLO, Ferreira MA. Feeding in adolescence and relationships with adolescent health. Florianópolis, Texto Context Enferm 2014; 23(4): 1095-103.

3. Pearson N, Ball K, Crawford D. Predictors of changes in adolescents' consumption of fruits, vegetables and energydense snacks. Australia. British J Nutr 2011; 105: 795-803.

4. Oliveira BL, Siqueira CF, Cordeiro KL, Guerra MAA, Diniz $A S$, Neto ACB. Adolescent's profile with overweight and obesity from municipal school system. Rev Enferm 2011; 
5(2): 205-212.

5. Brazil. Ministry of Health. Secretariat of Health Care. Department of Basic Attention. PSE / Ministry of Health Instruction. Department of Health Care. Department of Basic Attention - Brasilia DF; 2011.

6. Teixeira AS, Philippi ST, Leal GVS, Araki EL, Estima CCP, Guerreiro RER. Replacement of meals for snacks in adolescentes. Rev Paul Pediatr 2012; 30(3): 330-337.

7. Andrade SSCA, Yokota RTC, Sá NNB, Silva MMA, Araújo $W N$, Mascarenhas MDM, et al. Association between physical violence, consumption of alcohol and other drugs, and bullying among Brazilian adolescents. Cad Saude Publica 2012; 28(11): 1725-1736.

8. Pacheco SSM. The eating habit as a culturally produced behavior. In: Freitas MCS, Fontes GAV, Oliveira N, organizers. Writings and narratives about food and culture. Salvador: Edufba. 2008.

9. Araki EL, Philippi ST, Martinez MF, Estima CCP, Leal GVS, Alvarenga MS. Standard of meals performed by adolescents attending technical schools in São Paulo. Rev Paul Pediatr 2011; 29 (2): 164-170.

10. Martins APB, Levy RB, Claro RM, Moubarac JC, Monteiro $C A$. Increased contribution of ultra-processed food products in the Brazilian diet (1987-2009). Rev Saúde Pública 2013; 47(4): 1-10.

11. Bielemanni RM, Motta JVS, Minten GC, Horta BL, Gigante $D P$. Consumo de alimentos ultraprocessados e impacto na dieta de adultos jovens. Rev Saúde Pública 2015; 49:28. DOI:10.1590/S0034 8910.2015049005572.

12. Louzada ML, Baraldi LG, Steele EM, Martins AP, Canella DS, Moubarac JC, et al. Consumption of ultra-processed foods and obesity in Brazilian adolescents and adults. Preventive Medicine 2015; 81: 9-15.

13. Bigornia SI, LaValley MP, Moore LL, Northstone K, Emmett $P$, Ness AR, Newby PK. Dairy Intakes at Age 10 Years Do Not Adversely Affect Risk of Excess Adiposity at 13 Years. I Nutr 2014; 144(7): 1081-1090.

14. Juul F, Hemmingsson E. Trends in consumption of ultraprocessed foods and obesity in Sweden between 1960 and 2010. Public Health Nutrition 2015; 18(17): 30963107.
15. Jaime $P C$, Stopa $S R$, Oliveira $T P$, Vieira $M L$, Szwarcwald $C L$, Malta DC. Prevalência e distribuição sociodemográfica de marcadores de alimentação saudável, Pesquisa Nacional de Saúde, Brasil 2013. Epidemiol. Serv. Saúde 2015 AprJun; 24(2): 1.

6. Silva DCA, Frazão IS, Osório MM, Vasconcelos MGL. Perception of adolescents about the practice of healthy eating. Ciência \& Saúde Coletiva 2015; 20(11): 3299-3308.

17. Tavares LF, Castro IRR, Levy RB, Cardoso LO, Passos MD, Brito FSB. Relative validity of food practice indicators from the National School Health Survey among adolescents from Rio de Janeiro, Brazil. Rio de Janeiro. Cad Saude Publica 2014; 30(5): 1029-1041.

18. Silva JRM, Schmitz BAS, Rodrigues MLCF, Gabriel CG. Promotion of healthy eating at schools in the Federal District of Brazil. Rev Nutr 2013; 26(2): 145-158.

19. Souza GB, Junqueira SR, Araujo ME, Botazzo C. Health practices: subjective assessment of adolescents. Rio de Janeiro, Saúde em Debate 2012; 36(95): 562-571.

20. Oliveira JS, Barufaldi LA, Abreu GA, Leal VS, Brunken GS, Vasconcelos SML, et al. ERICA: use of screens and consumption of meals and snacks by adolescents. Rev Saude Publica 2016; 50(sup/ 1): 7s.

21. Silva FMA, Smith-Menezes A, Duarte MFS. Fruit and vegetable consumption associated with other risk behaviors among adolescents in Northeast Brazil. Rev Paul Pediatr 2016; 34 (3): 309-315.

22. Leal VS, Lira PIC, Menezes RCE, Oliveira JS, Costa EC, Andrade SLLS. Malnutrition and overweight in children and adolescents: a review of Brazilian studies. Rev Paul Pediatr 2012; 30(3): 415-422.

23. Conceição SIO, Santos CJN, Silva AAM, Silva JS, Oliveira TC. Food consumption of schoolchildren in the public and private education networks in São Luís, Maranhão. Campinas, Rev Nutr 2010; 23(6): 993-1004.

24. Schneider BC, Duro SMS, Assunção MCF. Meat consumption by adults in southern Brazil: a population-based study. Ciência \& Saúde Coletiva 2014; 19(8): 3583-3592.

25. Ferreira $A B H$. Miniaurélio: o dicionário da língua portuguesa. 8. ed. Curitiba: Positivo, 2010. 895 p. ISBN 978-85-385-4240-7. 